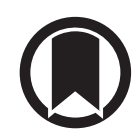

CrossMark

\title{
CSF1R inhibition prevents radiation pulmonary fibrosis by depletion of interstitial macrophages
}

\author{
Lydia Meziani $^{1,2}$, Michele Mondini ${ }^{1,2}$, Benoît Petit ${ }^{3}$, Alexandre Boissonnas ${ }^{4,5}$, \\ Vincent Thomas de Montpreville ${ }^{2,6}$, Olaf Mercier ${ }^{2,7}$, Marie-Catherine Vozenin (103,9 \\ and Eric Deutsch ${ }^{1,2,8,9}$
}

Affiliations: ${ }^{1}$ Gustave Roussy, Université Paris-Saclay, Inserm U1030, Villejuif, France. ${ }^{2}$ Labex LERMIT, DHU TORINO, SIRIC SOCRATE. ${ }^{3}$ Radio-Oncology Laboratory, Dept of Radiation Oncology/DO/CHUV, Lausanne University Hospital, Lausanne, Switzerland. ${ }^{4}$ INSERM, U1135, F-75013, Paris, France. ${ }^{5}$ Université Pierre et Marie Curie, Faculté de médecine, Paris, France. 'Service d'Anatomie Pathologique, Hôpital Marie Lannelongue, Le Plessis Robinson, France. ${ }^{7}$ Service de Chirurgie thoracique, Hôpital Marie Lannelongue, Le Plessis Robinson, France. ${ }^{8}$ Gustave Roussy, Université Paris-Saclay, Departement de radiotherapie, Villejuif, France. ${ }^{9}$ These authors contributed equally.

Correspondence: Eric Deutsch, INSERM 1030 Molecular Radiotherapy Gustave Roussy, 114 rue Edouard Vaillant, 94805 Villejuif, France. E-mail: eric.deutschdgustaveroussy.fr; Marie-Catherine Vozenin, Laboratoire de recherche en Radio-Oncologie, CHUV, Rue du Bugnon 21, 1011 Lausanne, Switzerland. E-mail: mariecatherine.vozenindachuv.ch

@ERSpublications

A novel approach to improve the management of radiation-induced lung complications via CSF1R inhibition http://ow.ly/dfWA30h6jxW

Cite this article as: Meziani L, Mondini M, Petit B, et al. CSF1R inhibition prevents radiation pulmonary fibrosis by depletion of interstitial macrophages. Eur Respir J 2018; 51: 1702120 [https://doi.org/10.1183/ 13993003.02120-2017].

ABSTRACT Radiation-induced lung fibrosis (RIF) is a delayed side-effect of chest radiotherapy, frequently associated with macrophage infiltration.

We aimed to characterise the role of pulmonary macrophages in RIF using human lung biopsies from patients receiving radiotherapy for thorax malignancies and a RIF model developed in C57BL/6 mice after 16-Gy thorax irradiation.

High numbers of macrophages (both interstitial and alveolar) were detected in clinical and preclinical RIF. In the preclinical model, upregulation of T-helper (Th)2 cytokines was measured, whereas Th1 cytokines were downregulated in RIF tissue lysate. Bronchoalveolar lavage demonstrated upregulation of both types of cytokines. At steady state, tissue-infiltrating macrophages (IMs) expressed 10-fold more arginase (Arg)-1 than alveolar macrophages (AMs), and a 40-fold upregulation of Arg-1 was found in IMs isolated from RIF. IMs, but not AMs, were able to induce myofibroblast activation in vitro. In addition, whereas depletion of AMs using Clodrosome didn't affect RIF score, depletion of IMs using a clinically available colony-stimulating factor receptor-1 (CSF1R) neutralising antibody was antifibrotic.

These findings suggest differential contributions of alveolar versus interstitial macrophages in RIF, highlighting the fibrogenic role of IMs. The CSF1/CSF1R pathway was identified as a new therapeutic target to inhibit RIF.

This article has supplementary material available from erj.ersjournals.com

Received: March 102017 | Accepted after revision: Dec 042017

Support statement: This work has received funding from Institut National du Cancer (INCa) and Eli Lilly. Funding information for this article has been deposited with the Crossref Funder Registry.

Conflict of interest: Disclosures can be found alongside this article at erj.ersjournals.com

Copyright OERS 2018 


\section{Introduction}

Fibrosis is a severe side-effect of radiotherapy [1], resulting in the accumulation of extracellular matrix (ECM) and organ loss of function. It has long been known that myofibroblasts are key players in the maintenance of fibrosis through the secretion of collagens [2] and the activation of the transforming growth factor (TGF)- $\beta 1 /$ Smad pathway, perpetuating the expression of downstream fibrogenic factors, such as PAI-1 [3]. However, more recent studies have reported a role for macrophages as critical regulators of fibrosis [4] and the involvement of M2-polarised macrophages in various models of fibrosis [5, 6] has been described. Macrophage polarisation is controlled by several factors, including CSF1R signalling, which was shown to be critical for shaping the M1/M2 macrophage phenotype $[7,8]$. The role of macrophage polarisation has been studied in irradiated solid tumours [9], and high radiation doses ( $>8 \mathrm{~Gy}$ ) have been shown to promote M2 polarisation [10], whereas lower doses $(<2 \mathrm{~Gy})$ are able to reprogramme macrophages towards the M1 phenotype which favours tumour elimination [11]. These recent data suggest that high versus low doses of radiotherapy trigger differential activation of macrophages in tumours. However, the role of macrophages in radiation-induced lung fibrosis (RIF) is less well characterised. Thus, we aimed to investigate the role of pulmonary macrophages using a well-characterised experimental model of lung fibrosis [12] induced by a single high dose of thorax irradiation, comparable to the doses currently administered during stereotactic body radiotherapy treatment. Because the tolerance of normal lung to irradiation is critical, lungs were selected as a reference organ where the macrophage contribution is pivotal [13]. Indeed, macrophage alveolitis has been consistently associated with RIF. Our results show that clinical and preclinical RIF are highly infiltrated by macrophages. Cytokine profiling of RIF tissue lysate showed upregulation of $\mathrm{T}$ helper (Th)2 cytokines, whereas Th1 cytokines were downregulated. However, bronchoalveolar lavage (BAL) showed upregulation of both types of cytokines. Consistently, interstitial macrophages (IMs) decreased the membrane expression of the pro-inflammatory marker Icam1 and increased the membrane expression of the anti-inflammatory marker CD206, whereas both markers (Icam1 and CD206) were increased at the membrane of alveolar macrophages (AMs). The differential phenotype between IMs and AMs was supported by expression analysis of genes involved in macrophage activation. At steady state, IMs expressed 10-fold more arginase (Arg)-1 than AMs. Furthermore, a 40-fold upregulation of Arg-1 was detected in IMs isolated from RIF lungs. Finally, using depletion experiments, we highlighted a differential contribution of IMs versus AMs to RIF, as depletion of IMs using a neutralising antibody against colony-stimulating factor receptor-1 (CSF1R) showed antifibrotic action, whereas depletion of AMs using clodronate liposome (Clodrosome; Interchim, Montluçon, France) had no effect.

\section{Materials and methods}

For more details on the materials and methods, refer to the online supplementary material.

\section{Human tissue samples}

Sample collection from the local tumour bank was performed, approved by the French Ministry of Research (AC-2016-2844). The study was approved by the local scientific and ethical board and waived the need for specific consent. Samples were collected from 10 patients operated in our department for thoracic malignancies between 2012 and 2016. Four patients had preoperative radiotherapy and received between 35 and 60 Gy before left pneumonectomy. In addition, six patients without radiotherapy were included; they underwent surgery and were used for control purposes.

\section{Animals and irradiation procedure}

Female C57BL/6J mice aged 12 weeks were purchased from Charles River Laboratories (Saint-Germain-Nuelles, France). The mice were immobilised through anaesthesia (2\% isoflurane) and locally irradiated at the thorax using a Varian Tube NDI 226 (X-ray machine; $250 \mathrm{Kev}$, tube current: $15 \mathrm{~mA}$, beam filter: $0.2 \mathrm{~mm} \mathrm{Cu}$ ), with a dose rate of $1.08 \mathrm{~Gy} \cdot \mathrm{min}^{-1}$. A single dose of $16 \mathrm{~Gy}$ was locally administered to the whole thorax.

Flow cytometry and fluorescence-activated cell sorting

F4/80-FITC (eBioscience, Paris, France), Gr1-PE (eBioscience), CD11c-PE-Cy7 (Biolegend, Ozyme, Montigny-le-Bretonneux, France), and CD11b-APC-Cy7 (Biolegend) were used to identify neutrophils $\left(\mathrm{Grl}^{\text {high }}\left(\mathrm{Ly}_{6 \mathrm{G}^{+}} \mathrm{Ly}^{-} \mathrm{C}^{-}\right)\right)$, monocytes $\left(\mathrm{Grl}^{+}\left(\mathrm{Ly}^{-} \mathrm{G}^{-} \mathrm{Ly}^{+} \mathrm{C}^{+}\right)\right)$, AMs $\left(\mathrm{CD} 11 \mathrm{c}^{+} \mathrm{CD} 11 \mathrm{~b}^{-} \mathrm{F} 4 / 80^{+}\right)$[14] and IMs (Gr1 $1^{-} \mathrm{CD}_{11 \mathrm{~b}^{+}} \mathrm{CD} 11 \mathrm{c}^{-/+} \mathrm{F} 4 / 80^{+}$) [15]. $\mathrm{Gr}^{-}$mononuclear phagocytes (MPs) were identified as $\mathrm{Gr} 1^{-}$ $\mathrm{CD} 11 \mathrm{~b}^{+} \mathrm{CD} 11 \mathrm{c}^{+} \mathrm{F} 4 / 80^{-}$. Icam1-Pacific blue (Biolegend) was used to identify M1-polarised macrophages and CD206-PerCpCy5.5 (Biolegend), to identify M2-polarised macrophages. CD115-PE (eBioscience) was used to examine CSF1R expression in AMs and IMs. The samples were analysed using FlowJo 10.0.7 
(FlowJo, Ashland, OR, USA). IMs and AMs were sorted using fluorescence-activated cell sorting and transferred in the culture medium.

\section{Encapsulated-clodronate liposome (Clodrosome) administration}

Approximately $60 \mu \mathrm{L}$ of Clodrosome was administered intranasally [16] 15 weeks after thoracic irradiation, every 3-4 days over 5 weeks for the duration of the experiment.

\section{Pulmonary macrophages inhibition using CSF1R monoclonal antibody}

15 weeks after thoracic irradiation, irradiated and control animals were treated with the neutralising anti-CSF1R monoclonal antibody (mAb) (CS7; Eli Lilly, Indianapolis, IN, USA) at $40 \mathrm{mg} \cdot \mathrm{kg}^{-1}$ (i.p.) three times a week for 5 weeks.

\section{Statistical analysis}

Statistical analyses were performed using GraphPad Prism 7 (GraphPad Software, San Diego, CA, USA). Two-way ANOVA was used to detect differences between irradiated and nonirradiated mice over time with subsequent Sidak multiple comparison tests. One-way ANOVA was used to detect differences among treated groups with subsequent Tukey multiple comparison test. A p-value $\leqslant 0.05$ was considered significant. Data were expressed as mean \pm SEM.

\section{Results}

\section{RIF is associated with an accumulation of pulmonary macrophages}

Irradiated lung parenchyma was obtained from the surgical samples from four patients who were treated between 1981 and 1998. They had received preoperative radiation; the total dose prescribed ranged from 35 to $60 \mathrm{~Gy}$ to treat their thoracic malignancies. Nonirradiated lung parenchyma was obtained from six patients who did not receive any radiotherapy. In contrast to control samples (figure 1a), samples from patients with RIF displayed thickening of alveolar walls and a decrease of alveolar air space, leading to a disappearance of alveolar structure (figure $1 \mathrm{~b}$ ). In order to characterise pulmonary macrophage infiltration in RIF area, CD163 staining was performed on irradiated and nonirradiated human lung tissue. We showed that $\mathrm{CD}_{163}{ }^{+}$pulmonary macrophages form clusters in alveoli of human tissue lung from irradiated patients (figure 1b, arrows). Interestingly, CD163 was highly expressed in fibrotic areas of irradiated human lung (figure $1 \mathrm{~b}$ and $\mathrm{c}$ ) in contrast to control lung tissue (figure 1a and c). The CD163 marker is described to be upregulated in M2-like macrophages [17], suggesting that human RIF could be associated with M2-like pulmonary macrophages. Given the very limited accessibility of human fibrotic and control lung tissues, we aimed at verifying this hypothesis by using a murine RIF model in order to deeply characterise the pulmonary myeloid subset infiltration during the development of RIF.
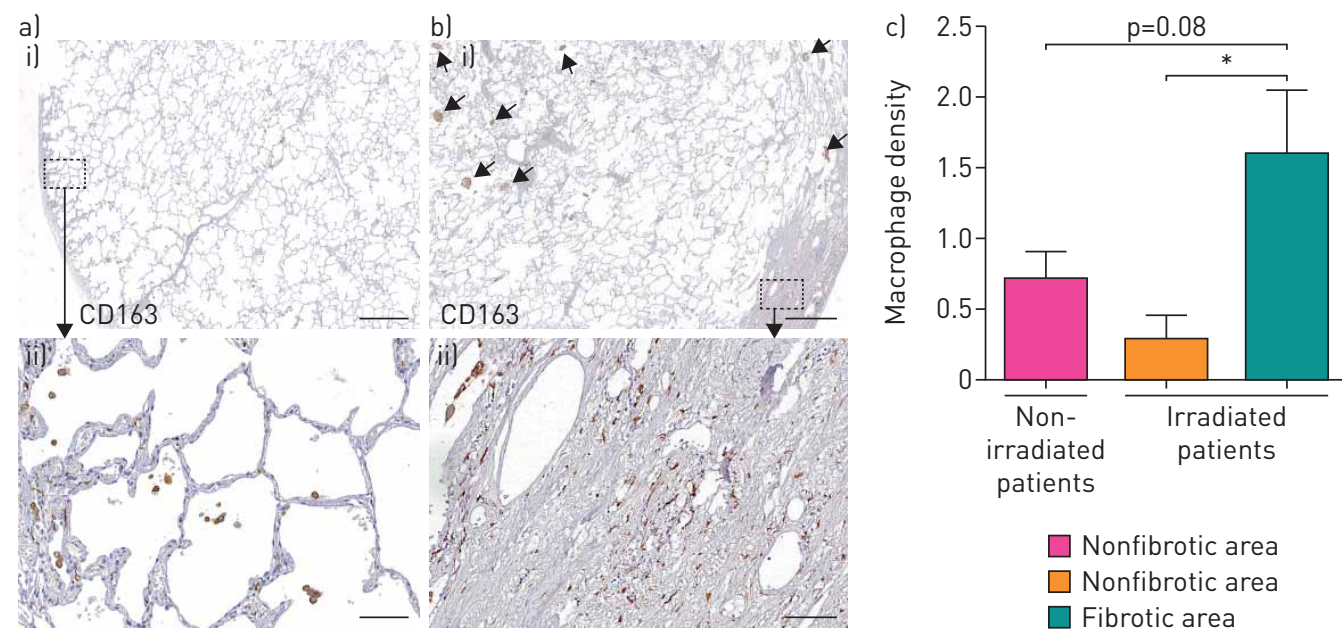

FIGURE 1 Human radiation-induced lung fibrosis is associated with high $\mathrm{CD}_{163^{+}}$macrophage infiltration. a) and b) Histological assessment of macrophage infiltration using anti-CD163 markers by DAB (3',3-diaminobenzidine) in paraffin-imbibed sections of nonirradiated and irradiated human lung parenchyma between 25 and $60 \mathrm{~Gy}$. Human biopsies were obtained from patients receiving thoracic radiotherapy between 1981 and 2012. i) Scale bars $=1 \mathrm{~mm}$; ii) scale bars $=100 \mu \mathrm{m}$. Arrows indicate macrophage clusters. c) Macrophage density quantification in irradiated $(n=5)$ patients and controls $(n=5)$. Data represent one

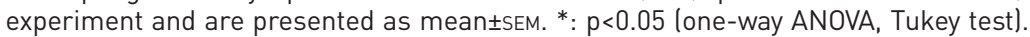


We first verified the development of lung fibrosis in the murine model of RIF described in the literature [12]. Cone beam computed tomography scans were performed at several time points after irradiation and showed a trend of increased lung density at day 15 after irradiation (figure 2a and b), suggesting an early inflammatory response in the lung. Interestingly, lung density returns to the basal rate by week 9 post-irradiation before a second increase from week 12 until week 19 after irradiation (figure 2a and b), suggesting the development of RIF. Monitoring of nonirradiated mice showed that lung density was stable over time (figure $2 \mathrm{c}$ and $2 \mathrm{~d}$ ). Histopathological examination of the lungs performed 20 weeks post-irradiation showed collagen deposition, as detected by the saffron (yellow) staining (figure 2e) and sirius red staining (online supplementary figure S1a) in irradiated lungs. Quantitative assessment of parenchymal fibrosis using automated histological image analysis software [18] showed a significant

a)
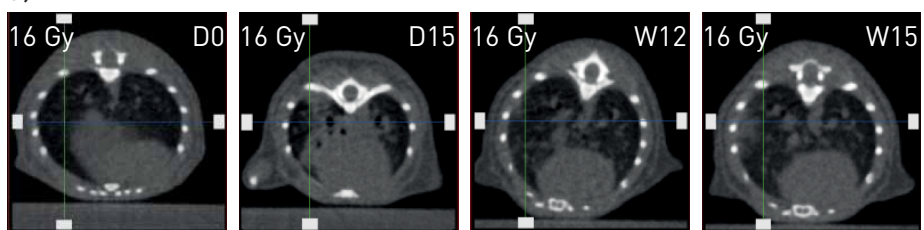

c)
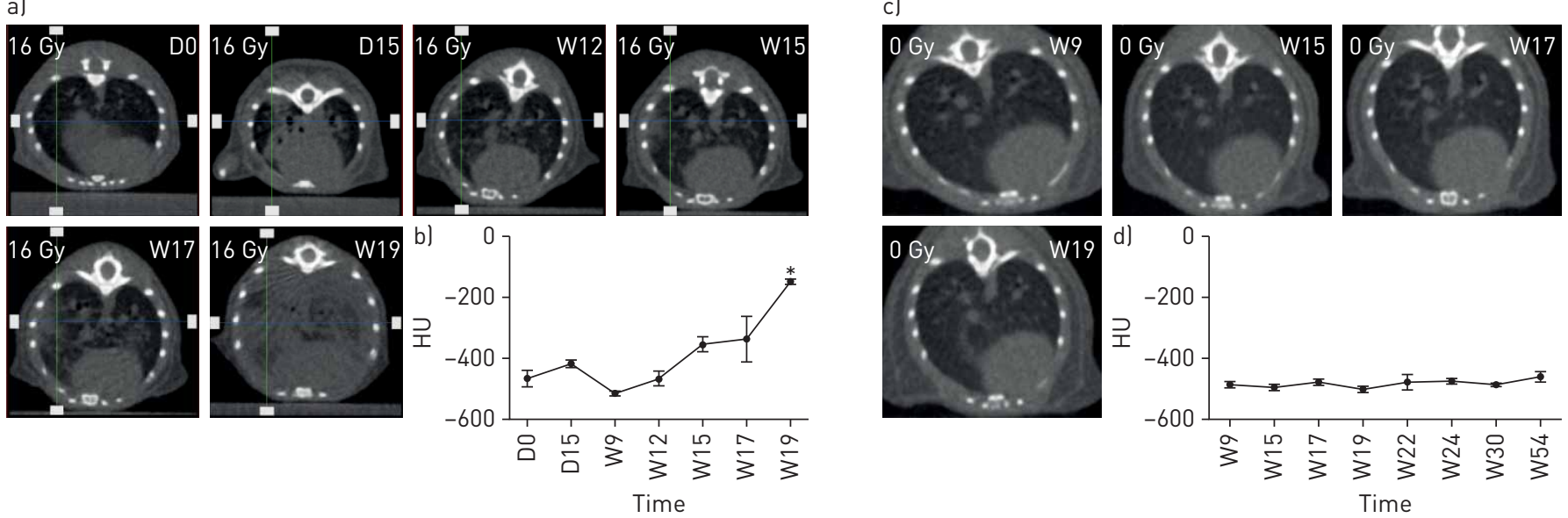

e)

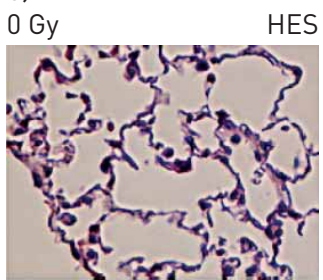

h)
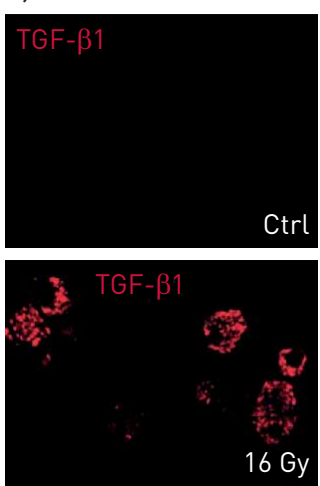

f) 20

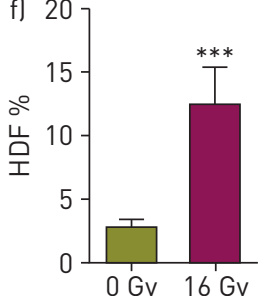

g)
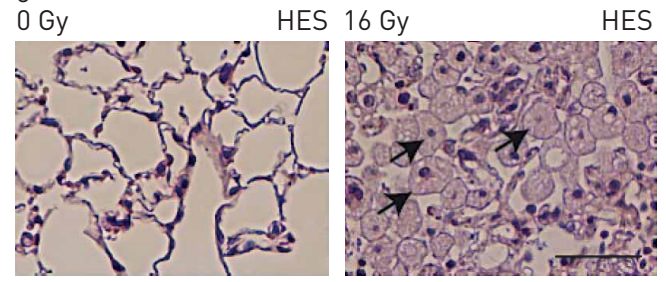

i)

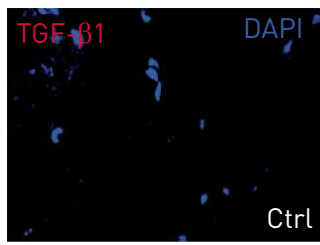

Ctrl

16 Gy

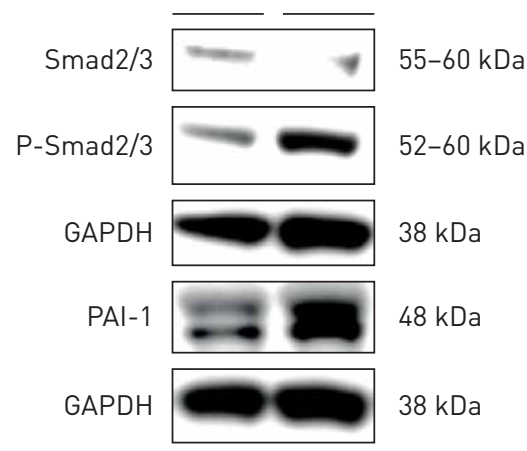

FIGURE 2 Preclinical radiation-induced lung fibrosis model and fibrosis quantification. a) Cone beam computed tomography (CBCT) scans of lung density of an irradiated C57BL/6 mouse at days (D) 0 and 15 and weeks (W) 12, 15, 17 and 19 after irradiation; b) lung density quantification in irradiated C57BL/6 mice ( $n=4) ;{ }^{*}$ : $p<0.05$ (one-way ANOVA, Dunnett test); c) CBCT scans of lung density of a nonirradiated C57BL/6 mouse over time; d) lung density quantification in nonirradiated C57BL/6 mice ( $n=5)$; e) representative images (haematoxylin-eosin-saffron (HES) staining) of lung fibrosis from irradiated $\mathrm{C} 57 \mathrm{BL} / 6$ mice $(n=3-6)$ versus age-matched controls, 20 weeks after irradiation; f) quantification of parenchymal fibrosis by the assessment of the high tissue density frequency (HDF) index in nonirradiated ( $n=10)$ and irradiated ( $n=8)$ C57BL/6 mice 20 weeks after irradiation. Data were obtained from three experiments and are presented as mean \pm SEM. ***: $p<0.001$ (t-test); g) representative images of macrophage infiltration in lung air spaces 20 weeks after irradiation. Arrows indicate macrophages. Scale bar=200 $\mu \mathrm{m}$; h) transforming growth factor (TGF)- $\beta 1$ immunofluorescence (red) and DAPI (4',6-diamidino-2-phenylindole) staining (blue) in lung tissue 20 weeks after irradiation. Scale bar $=15 \mu \mathrm{m}$; i) Western blot analysis of Smad2/3 Ser465/467 phosphorylation (p-Smad2/3) and Smad2/3, PAI-1 and GAPDH expression in lung tissue 20 weeks after irradiation. In b) and d) data were obtained from one experiment and are presented as mean \pm SEM. Ctrl: control. 
increase of high tissue density frequency (HDF) index in irradiated lungs versus age-matched controls (figure 2f). In addition, sirius red staining of collagen depositions was significantly increased after irradiation (online supplementary figure S1b). The fibrogenic pattern correlated with an important cell infiltrate (figure $2 \mathrm{~g}$ ), with a specific accumulation of TGF- $\beta 1$-expressing cells (figure $2 \mathrm{~h}$ ). Finally, RIF led
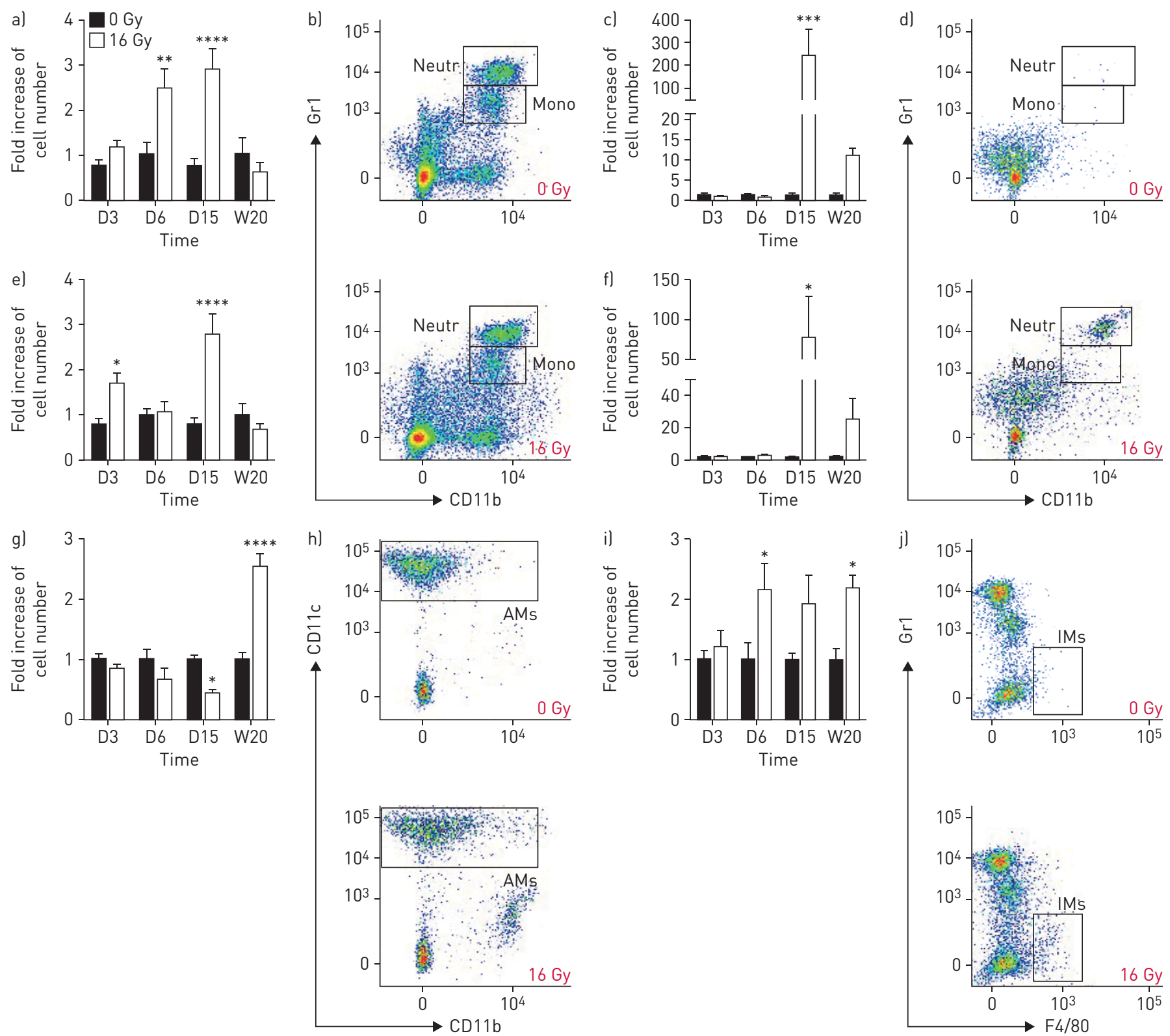

FIGURE 3 Inflammatory cell characterisation during thoracic radiation-induced injury. a) Fold increase of number of interstitial neutrophils within parenchyma during radiation injury in nonirradiated and irradiated C57BL/6 mice; b) gating strategy to identify neutrophils (Neutr; $\mathrm{Gr}^{+} \mathrm{CD}^{+} 1 \mathrm{~b}^{+}$) and monocytes (Mono; Gr1 ${ }^{\text {inter }} \mathrm{CD}_{1} 1 \mathrm{~b}^{+}$) within parenchyma of nonirradiated and irradiated mice 20 weeks after irradiation; c) fold increase of neutrophil number in bronchoalveolar lavage (BAL) during radiation injury in irradiated and nonirradiated mice; d) gating strategy to identify neutrophil $\left(\mathrm{Gr} 1^{\text {high }} \mathrm{CD} 11 \mathrm{~b}^{+}\right)$and monocytes $\left(\mathrm{Gr}^{+} \mathrm{CD}^{+} 1 \mathrm{~b}^{+}\right)$in BAL of nonirradiated and irradiated mice 20 weeks after irradiation; e) fold increase of number of interstitial monocytes within parenchyma during radiation injury in irradiated and nonirradiated mice; f) fold increase of monocyte number in BAL during radiation injury in irradiated and non-irradiated mice; g) fold increase in number of alveolar macrophages (AMs) during radiation injury in irradiated and nonirradiated mice; h) gating strategy to identify $A M s\left(C D 11 c^{+} C D_{11} b^{-}\right)$in nonirradiated and irradiated mice 20 weeks after radiation; i) fold increase of number of tissue-infiltrating macrophages (IMs) during radiation injury in irradiated and nonirradiated mice; j) gating strategy to identify $\mathrm{IMs}\left(\mathrm{F}_{4} / 80^{+} \mathrm{Gr}^{-}\right)$in nonirradiated and irradiated mice 20 weeks after irradiation. The absolute number was calculated using a fixed number of $10-\mu \mathrm{m}$ polybead carboxylate microspheres and expressed by the fold increase compared to the mean of control values at the same time point. Data were obtained from two independent experiments and presented as mean \pm SEM. D: day; W: week; ${ }^{*}: p<0.05 ;{ }^{* *}: p<0.01 ;{ }^{* *}$ : $p<0.001 ;{ }^{* * *}$ : $p<0.0001$. D3: 0 Gy, n=7-8; 16 Gy, n=8-9; D6: 0 Gy, n=7-8; 16 Gy, $n=7 ; D 15: 0$ Gy, $n=7-8 ; 16$ Gy, $n=8 ;$ week 20: $0 \mathrm{~Gy}, \mathrm{n}=7 ; 16 \mathrm{~Gy}, \mathrm{n}=8-10$ (two-way ANOVA, Sidak test). 
to increased PAI-1 expression and Smad2/3 phosphorylation, as shown by Western blot analysis (figure 2i).

Next, we characterised the cell infiltrate at different time points after irradiation in both the BAL and the lung interstitium (online supplementary figure S2). Neutrophil $\mathrm{Grl}{ }^{\text {high }}\left(\mathrm{Ly}_{6 \mathrm{G}}{ }^{+}\right.$) number increased significantly at 6 and 15 days post-irradiation in the parenchyma and returned to the basal rate at 20 weeks post-irradiation (figure $3 \mathrm{a}$ and $\mathrm{b}$ ). In the alveolar space (BAL), neutrophil infiltration was detectable only by day 15 and remained high at 20 weeks post-irradiation (figure $3 \mathrm{c}$ and $\mathrm{d}$ ). A transient $\mathrm{Gr}^{+}$monocyte infiltration in the parenchyma was detectable at day 3 after irradiation (figure $3 \mathrm{e}$ and $\mathrm{b}$ ). A second peak of infiltration was detectable by day 15 , correlating with an infiltration in the alveolar space, as observed for neutrophils (figure $3 \mathrm{f}$ and $\mathrm{d}$ ), and $\mathrm{Gr}^{+}$monocytes further accumulated in the alveolar space after 20 weeks. The number of $\mathrm{Gr}^{-}$MPs did not change over time in the parenchyma or in the BAL (data not shown).

Radiation induced a progressive decrease in the number of $\mathrm{AMs}\left(\mathrm{CD} 11 \mathrm{~b}^{-} \mathrm{CD} 11 \mathrm{c}^{+} \mathrm{F} 4 / 80^{+}\right)$present in the BAL until day 15 (figure 3g). Few AMs were harvested from the digested lung after extensive lavage of the bronchoalveolar space, suggesting a stronger adherence of AMs to the alveolar epithelium. The global accumulation of AMs was associated with an increased proportion of CD11b-expressing AMs. At 20 weeks post-irradiation, AMs recovered and were outnumbered (figure $3 \mathrm{~g}$ and $\mathrm{h}$ ), and at this time point up to $30 \%$ of $\mathrm{AMs}$ were $\mathrm{CD}_{11 \mathrm{~b}^{+}}$(online supplementary figure S3).

Finally, IMs $\left(\mathrm{CD} 11 \mathrm{c}^{-/+} \mathrm{CD} 1 \mathrm{~b}^{+} \mathrm{F} 4 / 80^{+}\right)$increased significantly at 6 days and 20 weeks post-irradiation in the lung parenchyma (figure $3 \mathrm{i}$ and $\mathrm{j}$ ) and were never detected in the BAL (data not shown), demonstrating that this subset resides exclusively in the lung interstitium.

We concluded that RIF is associated with a progressive infiltration and accumulation of inflammatory cells (monocytes and neutrophils) into the alveolar space during the early phase of RIF development and that it persists later on with lung fibrosis and increased accumulation of lung AMs and IMs during the late phase.

RIF is associated with a differential phenotype of AMs and IMs

Icam1 (as a pro-inflammatory marker) and CD206 (as an anti-inflammatory marker) membrane expression, as well as gene profiling was investigated in IMs and AMs. The number of Icam $1^{+}$and CD206 ${ }^{+}$AMs increased at week 20 post-irradiation (figure $4 \mathrm{a}$ and b) and both markers were upregulated on AMs from RIF lungs (figure $4 \mathrm{c}$ and $\mathrm{d}$ ). Microenvironment analysis by cytokine profiling of the acellular fraction of the BAL was consistent and showed that both pro-inflammatory Th1- and anti-inflammatory Th2-related cytokines (interleukin (IL)-2, IL-7, tumour necrosis factor- $\alpha$, IL-6 and IL-5) were induced after irradiation (figure $4 \mathrm{e}$ ).

IMs displayed bright expression of Icam1 and low expression of CD206 at steady state (figure 4f). The number of Icam $1^{+}$IMs transiently increased (figure $4 \mathrm{~g}$ ) and the level of expression of Icam 1 increased at day 6 post-irradiation and progressively returned to basal value at week 20 (figure $4 \mathrm{~h}$ ). During the late phase (week 20), an increased number of CD206 ${ }^{+}$IMs expressing a higher level of CD206 was observed (figure $4 \mathrm{i}$ and $4 \mathrm{j}$ ). Cytokine profiling of the IM microenvironment showed the downregulation of Th1-related cytokines, such as IL-1 $\alpha$ and IL-16, and the upregulation of TIMP-1, a Th2-related mediator (figure $4 \mathrm{k}$ ). Subsequently, we performed gene expression analysis on selected activation markers. One of the most striking results was obtained for Arg-1 mRNA level: at steady state Arg-1 expression was 10-fold greater in IMs than AMs and 40-fold greater in RIF-isolated IMs versus RIF-isolated AMs (figure 4l). In addition, Arg-1 mRNA level was induced by 400 -fold in IMs isolated from RIF, compared to IMs isolated from healthy lungs. Our results show that radiation impacts differentially the phenotype of AMs and IMs and that the development of RIF is associated with distinct local cytokine and chemokine microenvironments in the tissue and the BAL.

\section{Activated IMs are able to induce myofibroblastic activation and ECM production ex vivo}

The mechanism underlying the respective involvement of AMs and IMs in fibrogenesis was explored using ex vivo experiments. First, IMs and AMs were sorted from RIF tissue 15 weeks post-irradiation. Giemsa staining demonstrated that IMs and AMs from irradiated mice exhibited a foamy phenotype as compared with normal macrophages (figure 5a). RIF-isolated IMs showed enhanced expression of the CD206 marker and AMs enhanced expression of both CD206 and Icam1 markers (online supplementary figure S4), suggesting that AMs and IMs are already activated at this stage. Sorted IMs and AMs were subsequently co-cultured with normal fibroblasts using transwells, and myofibroblastic differentiation was examined using expression of $\alpha$-smooth muscle actin (SMA) and TGF- $\beta 1$. Induction of TGF- $\beta 1$ in fibroblasts was observed when fibroblasts were co-cultured with RIF-isolated IMs (figure 5b), but not with RIF-isolated AMs. 

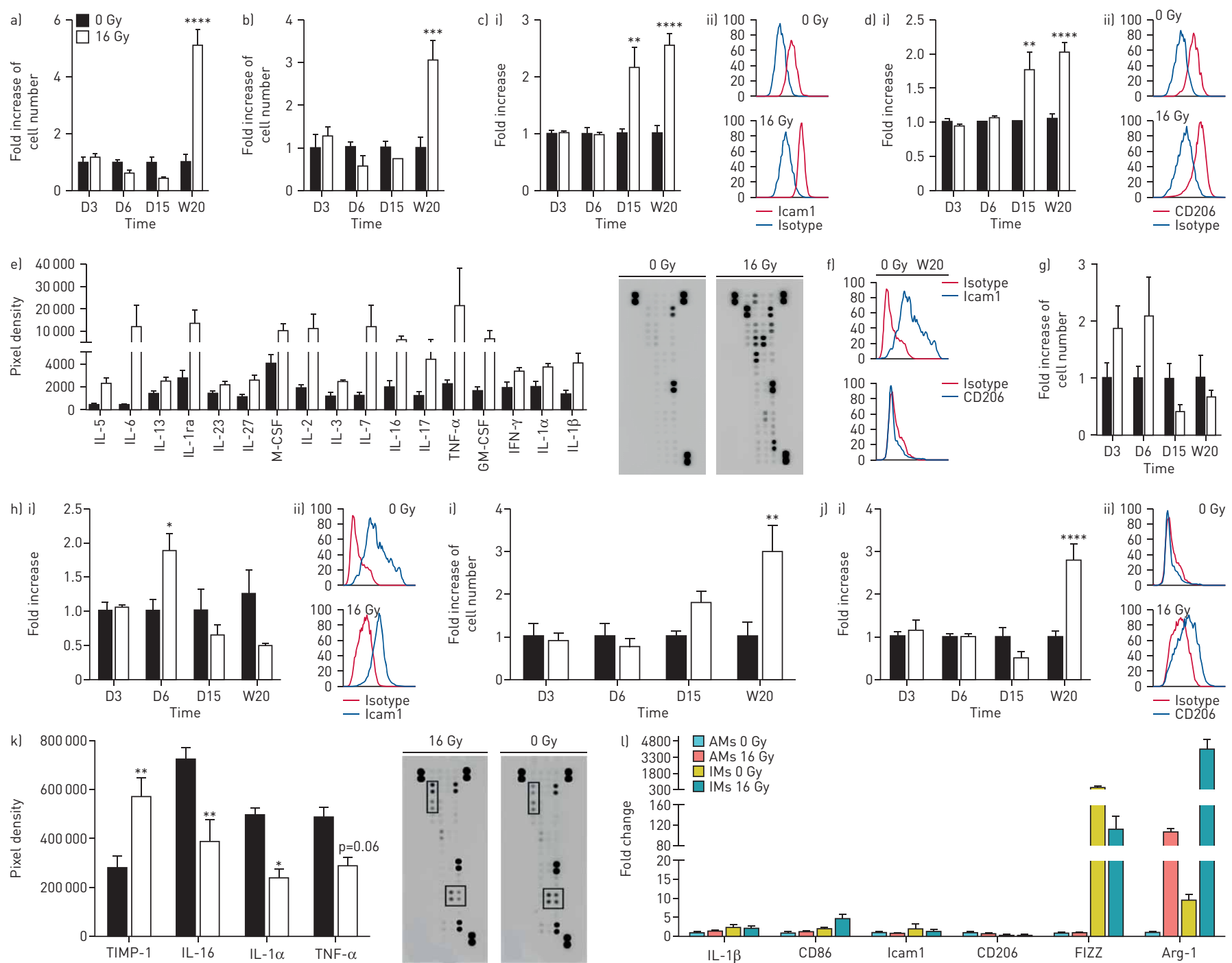

FIGURE 4 Phenotype characterisation of alveolar macrophages (AMs) and tissue-infiltrating macrophages (IMs) after thoracic irradiation. Fold increase of numbers of a) Icam $1^{+} \mathrm{AMs}$ and b) CD206 ${ }^{+} \mathrm{AMs}$ during radiation injury; c) i) fold increase of membrane $\Delta \mathrm{MFI}$ (mean fluorescence intensity of antibody/mean fluorescence of isotype control) of Icam1 in AMs during radiation injury; ii) representative single-parameter histograms of AMs stained with Icam1 marker in nonirradiated and irradiated mice at week 20 after irradiation; d) il fold increase of membrane $\Delta$ MFI of CD206 in AMs during radiation injury; ii) representative single-parameter histograms of AMs stained with CD206 marker in nonirradiated and irradiated mice at week 20 after irradiation; e) acellular fraction of bronchoalveolar lavage (BAL) analysed using cytokine arrays at week 20 post-irradiation and densitometric analysis of signals and representative blot; $\mathrm{f}$ ) representative single-parameter histograms of tissue-infiltrating macrophages (IMs) stained with Icam1 and CD206 marker in nonirradiated mice at week 20 after irradiation (0 Gy); g) fold increase of Icam ${ }^{+}$IM number during radiation injury; h) i) fold increase of membrane $\triangle \mathrm{MFI}$ of Icam1 in IMs during radiation injury; ii) representative single-parameter histograms of IMs stained with Icam1 marker in nonirradiated and irradiated mice at week 20 after irradiation; i) fold increase of CD206 ${ }^{+}$IM number during radiation injury; j] i) fold increase of membrane $\triangle M F I$ of CD206 in IMs during radiation injury; ii) representative single-parameter histograms of IMs stained with CD206 marker in nonirradiated and irradiated mice at week 20 after irradiation; k) lung tissue was digested and proteome analysis was performed using cytokine arrays at week 20 post-irradiation and densitometric analysis of signals was performed, with representative blots. The absolute number was calculated using a fixed number of $10-\mu \mathrm{m}$ polybead carboxylate microspheres and expressed by the fold increase compared to the mean of control values. Data were obtained from two independent experiments and presented as mean \pm SEM. $D$ : day; W: week; IL: interleukin; M-CSF: macrophage colony-stimulating factor; TNF: tumour necrosis factor; GM-CSF: granulocyte-macrophage colony-stimulating factor; IFN: interferon. D3: 0 Gy, n=8; 16 Gy, n=8-9; D6: 0 Gy, n=7-9; 16 Gy, $n=7 ; D 15: 0$ Gy, $n=8 ; 16$ Gy, n=8; W20: 0 Gy, $n=7$; $16 \mathrm{~Gy}, \mathrm{n}=8-10$. In e and k) $\mathrm{n}=3$ in each group. ${ }^{*}: \mathrm{p}<0.05 ;{ }^{* *}: \mathrm{p}<0.01 ;{ }^{* * *}: \mathrm{p}<0.001 ;{ }^{* * * *}$ : $\mathrm{p}<0.0001$ (two-way ANOVA, Sidak test). L) Fold change of mRNA of M1 and M2 markers in AMs and IMs at 20 weeks after irradiation. Data were obtained from one experiment and presented as mean \pm SD of technical replicates. 0 Gy: pooled samples from 9 mice; 16 Gy: pooled samples from 7 mice.

In addition, IMs and AMs were sorted from normal mouse lung and activated in vitro for $24 \mathrm{~h}$ using recombinant interferon- $\gamma$ to induce classical activation and recombinant IL-13/-4 to induce alternative activation. Activated AMs and IMs were then co-cultured with normal pulmonary fibroblasts without the activating mediators. The fibroblasts co-cultured with IL-13/-4-activated IMs overproduced ECM components (figure $5 \mathrm{c}$ ) and increased their $\alpha$-SMA expression (figure $5 \mathrm{~d}$ and e), whereas activated AMs 
a)

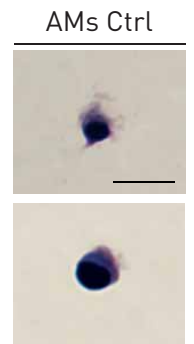

c)

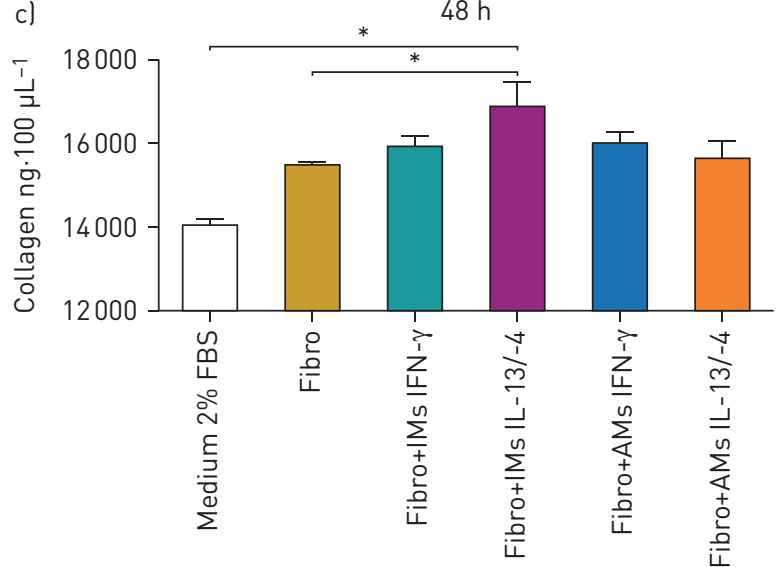

d)

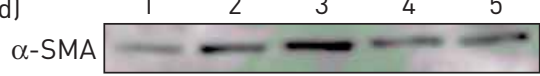

Vinculin

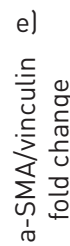
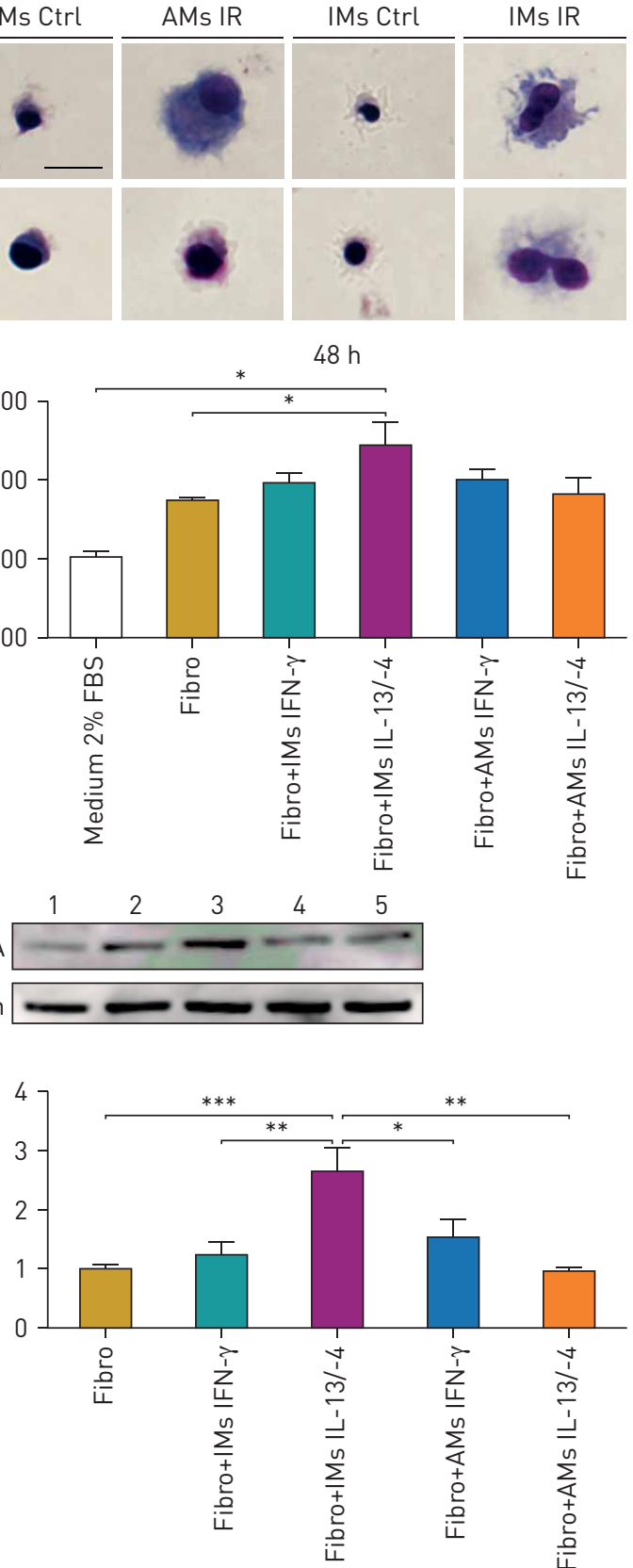

$48 \mathrm{~h}$

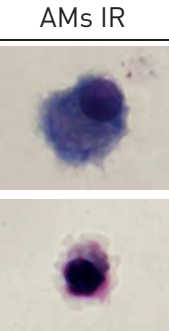

b)
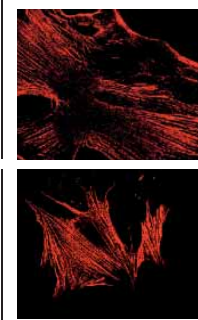

Fibroblasts

$+$

AMs Ctrl
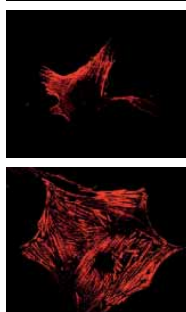

Fibroblasts

$\stackrel{+}{\text { IMs Ctrl }}$
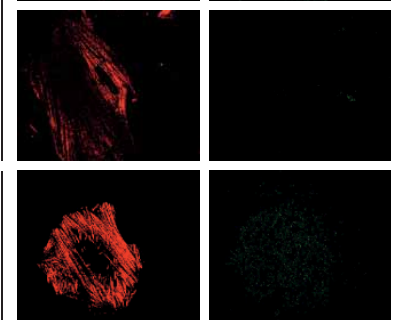

Fibroblasts

$\stackrel{+}{\text { AMs IR }}$
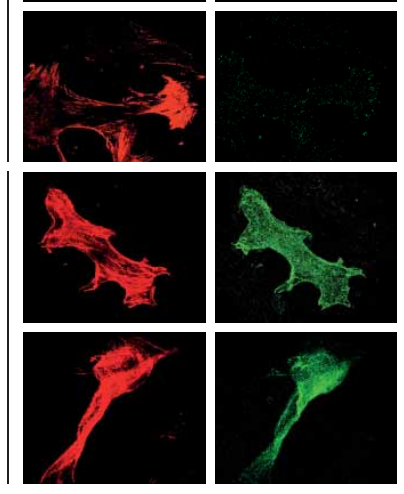

Fibroblasts

IMs IR
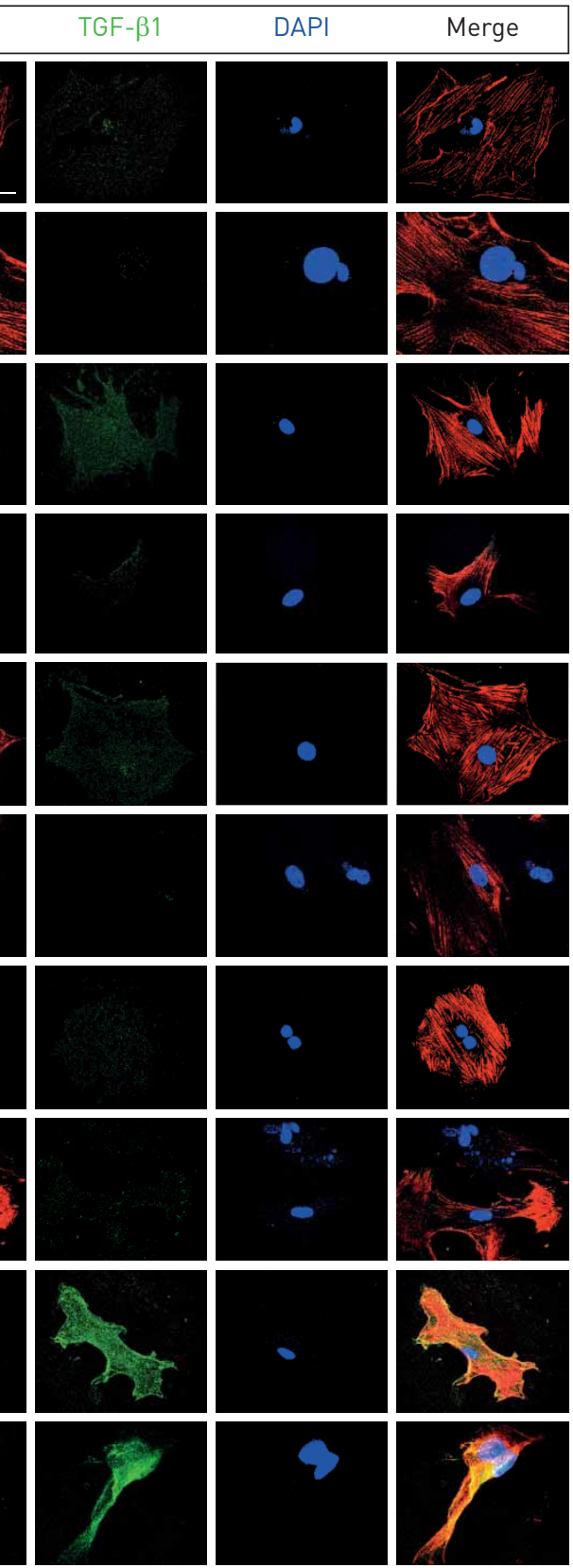

FIGURE 5 Normal fibroblasts and activated lung macrophage co-culture. a) May-Grünwald Giemsa staining of flow cytometry-sorted activated and nonactivated lung macrophages at week 15 post-irradiation. Scale bar=50 $\mu \mathrm{m}$. b) $\alpha$-smooth muscle actin (SMA) (red) and transforming growth factor (TGF)- $\beta 1$ immunofluorescence (green) and DAPI staining (blue), $24 \mathrm{~h}$ after fibroblast and macrophage co-culture (in triplicate). Scale bar $=20 \mu \mathrm{m}$. Data were obtained from one experiment. c) Collagen production quantification by Sircol assay in $100 \mu \mathrm{L}$ of fibroblast supernatant after $48 \mathrm{~h}$ of co-culture with in vitro activated alveolar macrophages (AMs)/tissue-infiltrating macrophages (IMs). d) Western blot of $\alpha$-SMA expression in whole-fibroblast lysate after $48 \mathrm{~h}$ of co-culture; 1) fibroblasts; 2) fibroblasts+IMs IFN- $\gamma$; 3) fibroblasts+IMs IL-13/-4; 4) fibroblasts + AMs IFN- $\gamma ; 5)$ fibroblasts+AMs IL-13/-4. e) Fold change quantification of $\alpha$-SMA expression/vinculin expression in whole-fibroblast lysate after $48 \mathrm{~h}$ of co-culture. Data were obtained from two independent experiments and presented as mean \pm SEM. $\mathrm{n}=6-7$. Ctrl: control; IR: irradiated; IFN: interferon; IL: interleukin. ${ }^{*}: \mathrm{p}<0.05 ;{ }^{* *}: \mathrm{p}<0.01{ }^{* * *}$ : $\mathrm{p}<0.001$ (one-way ANOVA, Dunnett test).

had no impact on ECM, $\alpha$-SMA production by normal fibroblasts. These results confirm the differential involvement of activated IMs versus AMs on the modulation of the phenotype of lung fibroblasts.

The specific depletion of AMs using Clodrosome has no effect on RIF

The intranasal administration of Clodrosome specifically depletes AMs but not IMs [16] and was used for the selective depletion of AMs at 15 weeks post-irradiation (figure 6a). This time point was chosen since 
a)

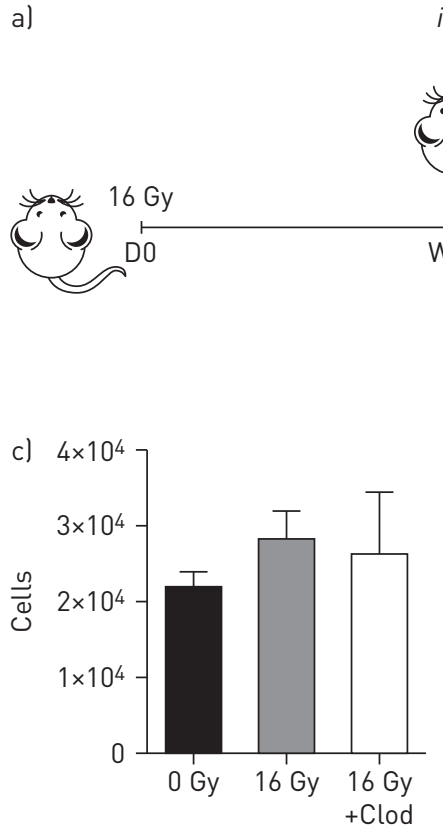

in.

Clodronate-

liposomes

(Clod)

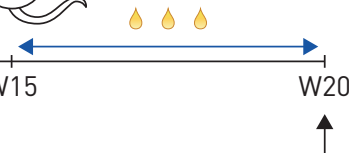

End of experiment

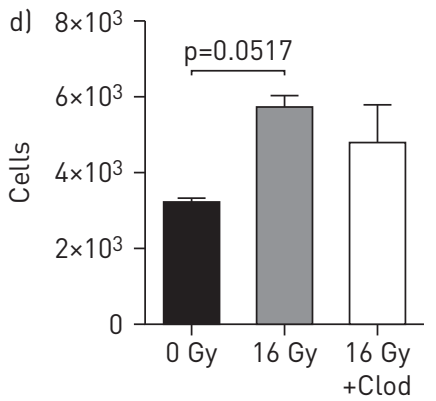

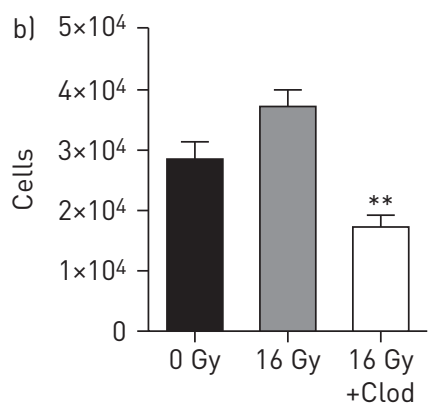

e)

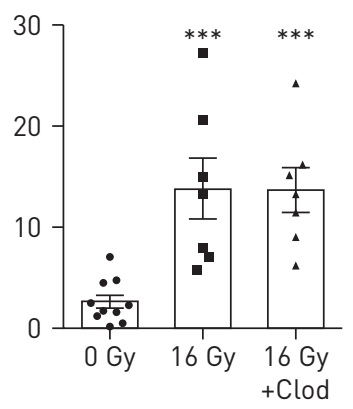

FIGURE 6 Specific targeting of alveolar macrophages (AMs) using clodronate-liposomes (Clod) after thoracic irradiation. a) AM depletion by intranasal (i.n.) administration of $60 \mu \mathrm{L}$ of Clod 15 weeks post-irradiation (16 Gy), in C57BL/6 mice. 20 weeks after irradiation, mice were sacrificed and half of the right lung was isolated and digested. Subsequently, lung tissue-derived cells were stained and analysed using flow cytometry. b) $A M$, cl monocyte and d) tissue-infiltrating macrophage number quantification using flow cytometry in different groups of treated animals. e) Quantification of parenchymal fibrosis by the assessment of the high tissue density frequency (HDF) index in treated and non-treated mice 20 weeks after irradiation. Calculation of absolute number was performed using a fixed number of non-fluorescent $10-\mu m$ polybead carboxylate microspheres. Data were obtained from two independent experiments and presented as mean \pm SEM. 0 Gy: n=7; 16 Gy: n=7; and 16 Gy+Clod: n=7. In e) 0 Gy: $n=10 ; 16$ Gy: n=7; 16 Gy+Clod: n=7. *: p<0.05; ${ }^{* *}: p<0.01 ;{ }^{* * *}: p<0.001$ (one-way ANOVA, Tukey test).

an increased number of $\mathrm{Icam}^{+} / \mathrm{CD} 206^{+} \mathrm{AMs}$ was observed at 15 weeks post-irradiation (online supplementary figure S4). The initial flow cytometry analysis confirmed the selective depletion of AMs through Clodrosome treatment (figure 6b). In contrast, monocytes (figure 6c), IMs (figure 6d) and $\mathrm{Gr}^{-}$ MPs (data not shown) were not affected by Clodrosome treatment. Clodrosome-mediated depletion of the AMs was associated with a high HDF fibrosis index at 20 weeks post-irradiation as well as in the irradiated mice not treated with Clodrosome (figure 6e).

The specific depletion of IMs but not AMs using CSF1R mAb blocks the development of RIF

Next, we attempted to deplete more broadly the lung macrophage subsets using an anti-CSF1R antibody (CS7). Mice were treated three times a week for 5 weeks starting at week 15 after thoracic irradiation (figure 7a). Accordingly, at 20 weeks post-irradiation we showed that the treatment with the CSF1R mAb specifically reduced IMs (figure $7 \mathrm{~b}$ ) and $\mathrm{Gr}^{-}$MPs (figure $7 \mathrm{c}$ ). No reduction in the number of $\mathrm{Gr}^{+}$ monocytes and AMs were observed at this time point (figure $7 \mathrm{~d}$ and e). Subsequently, we analysed CSF1R expression on AMs and showed that AMs, in contrast to IMs, express low levels of CSF1R (figure 7f), thus accounting for the lack of modulation of this population by CS7. Interestingly, anti-CSF1R treatment reduced more drastically the number of $\mathrm{CD}_{206}{ }^{+} \mathrm{IMs}_{\text {compared to Icam1 }}^{+}$IMs (figure $7 \mathrm{~g}$ and $\mathrm{h}$ ). This reduction was associated with a dramatic decrease of RIF (figure 7i and online supplementary figure S5) as well as a marked reduction of PAI-1 expression and Smad2/3 phosphorylation level (figure 7j).

Finally, we explored the possibility that pulmonary fibroblasts trigger the activation of lung macrophages in irradiated mice. Primary lung fibroblasts isolated from irradiated and nonirradiated mice were co-cultured with either normal AMs or normal IMs, and the supernatants were collected after $24 \mathrm{~h}$ of co-culture (online supplementary figure S6a). The supernatants produced from fibroblasts isolated from irradiated lungs + IMs contained higher amounts of CSF1 and monocyte chemoattractant protein-1 than the supernatants produced from irradiated fibroblasts + AMs or from nonirradiated fibroblasts + IMs (online supplementary figure S6b and c). These results suggest that irradiated fibroblasts can activate 


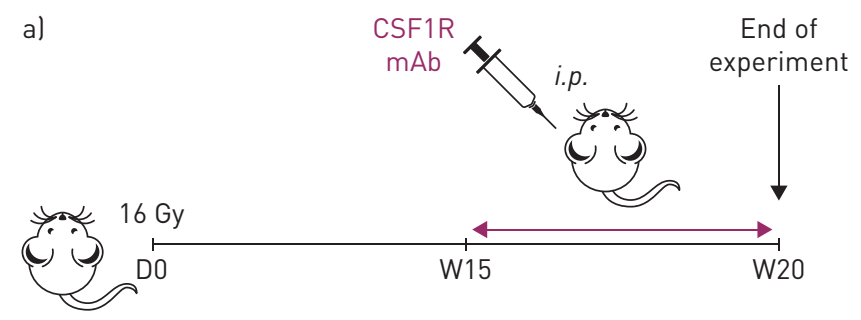

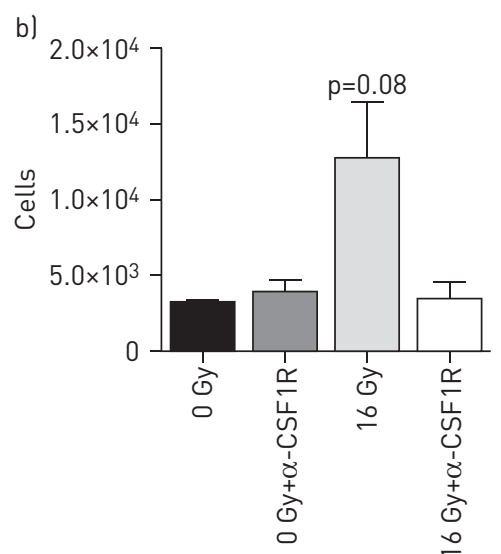
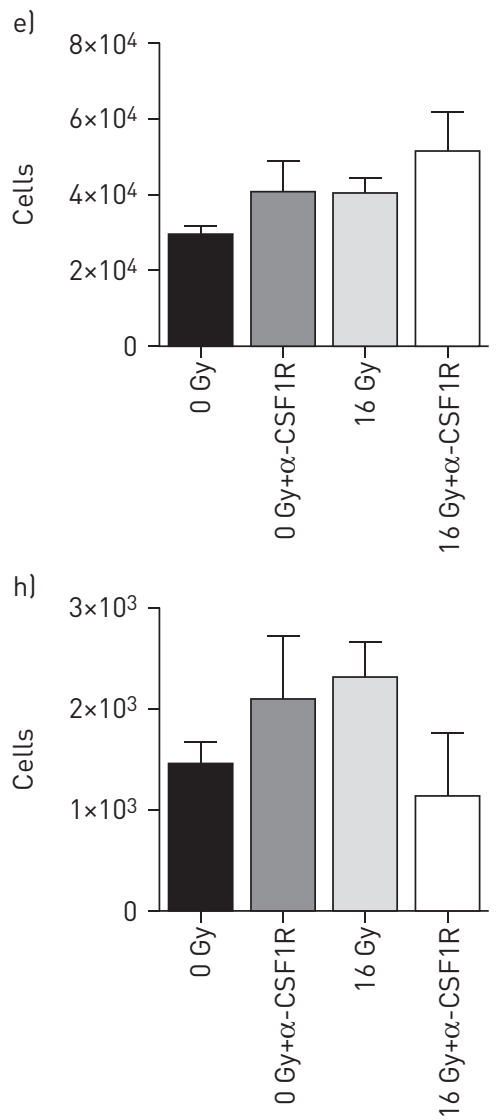
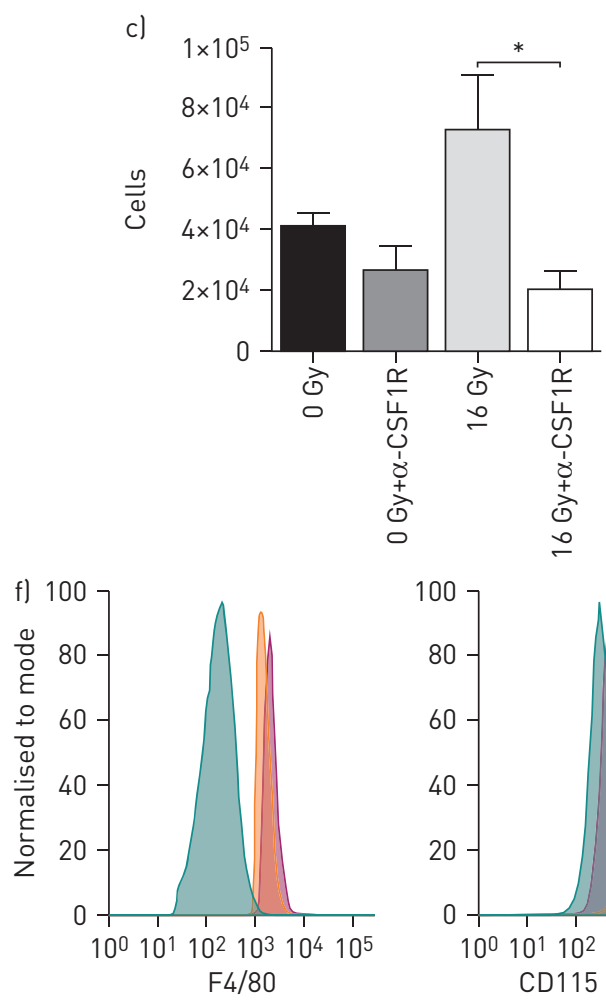

Isotype control

IMs

AMs

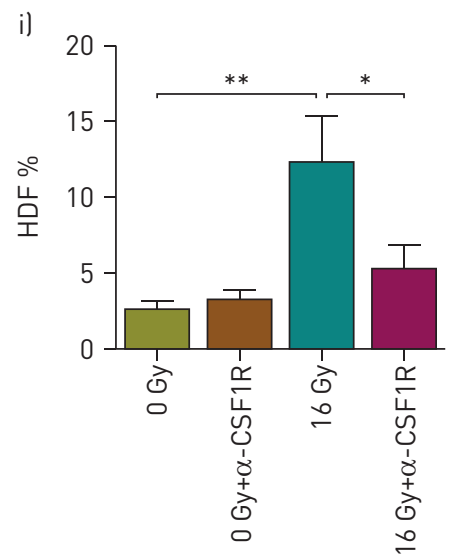

j) $16 \mathrm{~Gy}$ d)

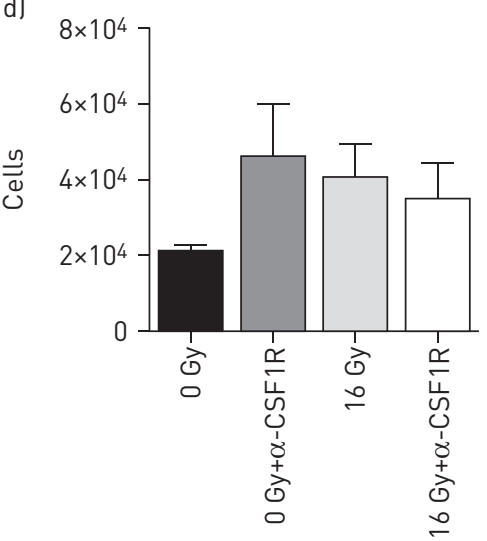

g)

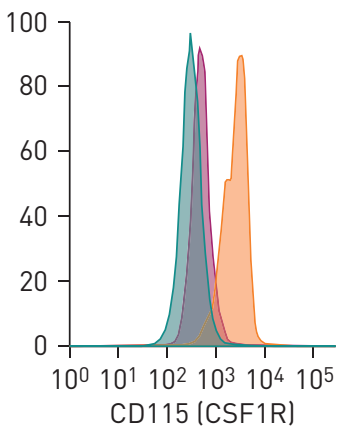
$\begin{array}{lllll}16 \text { G } & - & + & + & - \\ \alpha-C S F 1 R & - & - & + & +\end{array}$

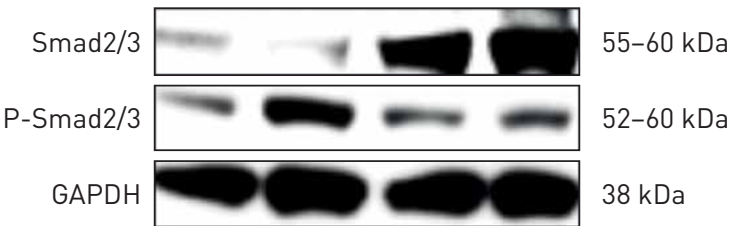

PAI-1

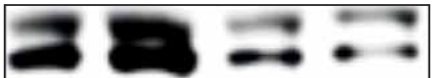

$48 \mathrm{kDa}$

GAPDH

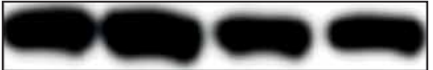

$38 \mathrm{kDa}$

FIGURE 7 Targeting of tissue-infiltrating macrophages (IMs) by anti-CSF1R after thoracic irradiation. C57BL/6 mice ( $\mathrm{n}=4-6)$ were irradiated at the thorax. a) CSF1R mAb administration for mice (i.p.) at 15 weeks post-irradiation (16 Gy). At 20 weeks post-irradiation mice were sacrificed. Right

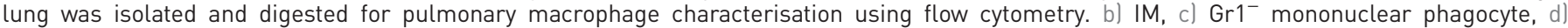
monocyte and e) alveolar macrophage (AM) number quantification in different groups of treated animals. f) Representative single-parameter histograms of IMs and AMs stained with F4/80 and CD115 (CSF1R). g) CD206 ${ }^{+}$IM and h) Icam1 ${ }^{+}$IM number quantification. i) Histological assessment of fibrosis score by determination of the high lung tissue density frequency (HDF) in treated and non-treated mice at 20 weeks after irradiation. Data are from three experiments, 0 Gy: $n=10 ; 0$ Gy+ $\alpha$-CSF1R: $n=9 ; 16$ Gy: $n=8 ; 16 G+\alpha-C S F 1 R: n=12$. j) Western blot analysis of Smad2/3 Ser465/467 phosphorylation (p-Smad2/3) and Smad2/3, PAl-1 and GAPDH expression in digested lung tissue at 20 weeks post-irradiation. Calculation of absolute number was performed using a fixed number of non-fluorescent $10-\mu \mathrm{m}$ polybead carboxylate microspheres. Data were obtained from one experiment and presented as mean \pm SEM. *: $p<0.05 ;{ }^{* *}$ : $p<0.01$ (one-way ANOVA, Tukey test). 
resident/recruited macrophages to favour the recruitment of inflammatory cells and the polarisation of the different subsets toward a profibrogenic profile.

We conclude that depletion of IMs, but not of AMs, by CSF1R mAb administration blocks RIF development in the lungs.

\section{Discussion}

A pivotal role for macrophages in the tumour response to radiotherapy was recently uncovered [19], with a possible role for alternatively activated macrophages in the release of pro-angiogenic factors that contribute to tumour resistance and/or regrowth post-irradiation [10]. In the present study, using the lungs as a model, we investigated the normal tissue response to radiotherapy and extensively characterised the contribution of pulmonary macrophages. The following conclusions were reached: 1) both human and preclinical RIF are associated with macrophage infiltration, suggesting an important role for pulmonary macrophages in RIF; 2) a fibrogenic dose of irradiation induces both an acute pro-inflammatory response occurring first in the parenchyma and then in the alveoli, and a late inflammatory response characterised by AMs and IMs with differential phenotype; 3) RIF-isolated IMs are suggested to induce fibrogenic process by induction of the myofibroblastic differentiation; these results are confirmed in vitro using IMs activated with IL-13/-4; and 4) specific depletion of IMs in RIF with CSF1R mAb, but not the depletion of AMs, exerted an anti-fibrotic action and CSF1R has been identified as a potent antifibrotic target.

The lungs are highly sensitive to irradiation and have been characterised as a major dose-limiting organ in radiotherapy. Both acute and delayed radiation-induced pulmonary toxicity have been described at the pathophysiological and cellular levels $[20,21]$. Clinically acute pulmonary toxicity was described almost 30 years ago and named "acute radiation pneumonitis" [21]. This acute syndrome is characterised by macrophage infiltration in air spaces and mononuclear inflammatory cells in alveolar walls [12]. Consistently, our preclinical model repeats these older results and shows a differential timing of inflammatory cell infiltration between the parenchyma and the alveolar space. Delayed pulmonary toxicity is clinically characterised by a chronic progressive pulmonary RIF [21]. The chronic stage is mainly characterised by a diffuse subpleural fibrosis, alveolar collapse or disappearance and their replacement by nonfunctional connective tissue [21] as shown in figure 1. In murine models, macrophage alveolitis was described as a hallmark of RIF in the lungs [12]. In summary, both human and murine RIF is associated with high macrophage infiltration and our hypothesis was that pulmonary macrophages were able to trigger the onset and maintenance of RIF.

Our results support this hypothesis and provide the first evidence for a differential role for AMs and IMs in the fibrogenic process. There is considerable evidence suggesting that AMs and IMs represent distinct cell populations [22]. Accordingly, we showed a differential gene expression at steady state between AMs and IMs and we describe for the first time a lower CSF1R membrane expression in AMs compared to IMs. Furthermore, unlike RIF-isolated AMs, RIF-isolated IMs lose Icam1 but enhance CD206 membrane expression and overexpressed Arg-1 mRNA. In addition, RIF biopsies from patients revealed a high infiltration by $\mathrm{CD} 163^{+}$macrophages described as alternatively activated macrophages [17]. This macrophage subtype has also been described in bleomycin-induced pulmonary fibrosis [23] and idiopathic pulmonary fibrosis [23], driven by specific cytokine patterns present in the microenvironment [24, 25]. Furthermore, we provide evidence that irradiated lung fibroblasts themselves can contribute to macrophage activation through the secretion of cytokines, such as CSF. Since RIF-isolated and IL-13/-4-activated IMs stimulate fibroblast differentiation into myofibroblasts. Thus, the signals seem to be bidirectional and a functional crosstalk between fibroblast and IMs is demonstrated in RIF, as described during prostate carcinoma progression [26]. Our findings identify a novel fibrogenic loop that involves and sustains interstitial macrophage and fibroblast activation, as illustrated in figure 8.

This scheme is consistent with the results obtained in other types of fibrosis $[13,27]$ and is the first to demonstrate that IMs have the potential to trigger specific features of RIF. These results thus support the relevance of an antimacrophage strategy as an antifibrotic therapy. We identified and showed that CSF1R was a therapeutic target. Indeed, given the preferential expression of CSF1R on IMs, the use of anti-CSF1R neutralising antibody resulted in a specific depletion of the profibrotic IMs, leaving unaffected the population of the AMs (see the model in figure 8) and reversed RIF. The clinical application of CSF1R inhibition in combination with radiotherapy extends beyond normal tissue toxicity since it has been described as a potent suppressor of tumour growth when combined with radiotherapy in a mouse prostate cancer model [28]. It suggests that targeting the CSF1/CSF1R axis using a CSF1R inhibitor can enhance the therapeutic index of radiotherapy, but further studies are required to validate this hypothesis in lung models. Many pharmaceutical companies are developing therapeutic tools targeting macrophages, and early clinical studies suggest that CSF1R inhibitors [29] have clinical utility. The anti-CSF1R antibody used in our study (CS7) is the murine counterpart of the human CS4 antibody currently in clinical trials for 


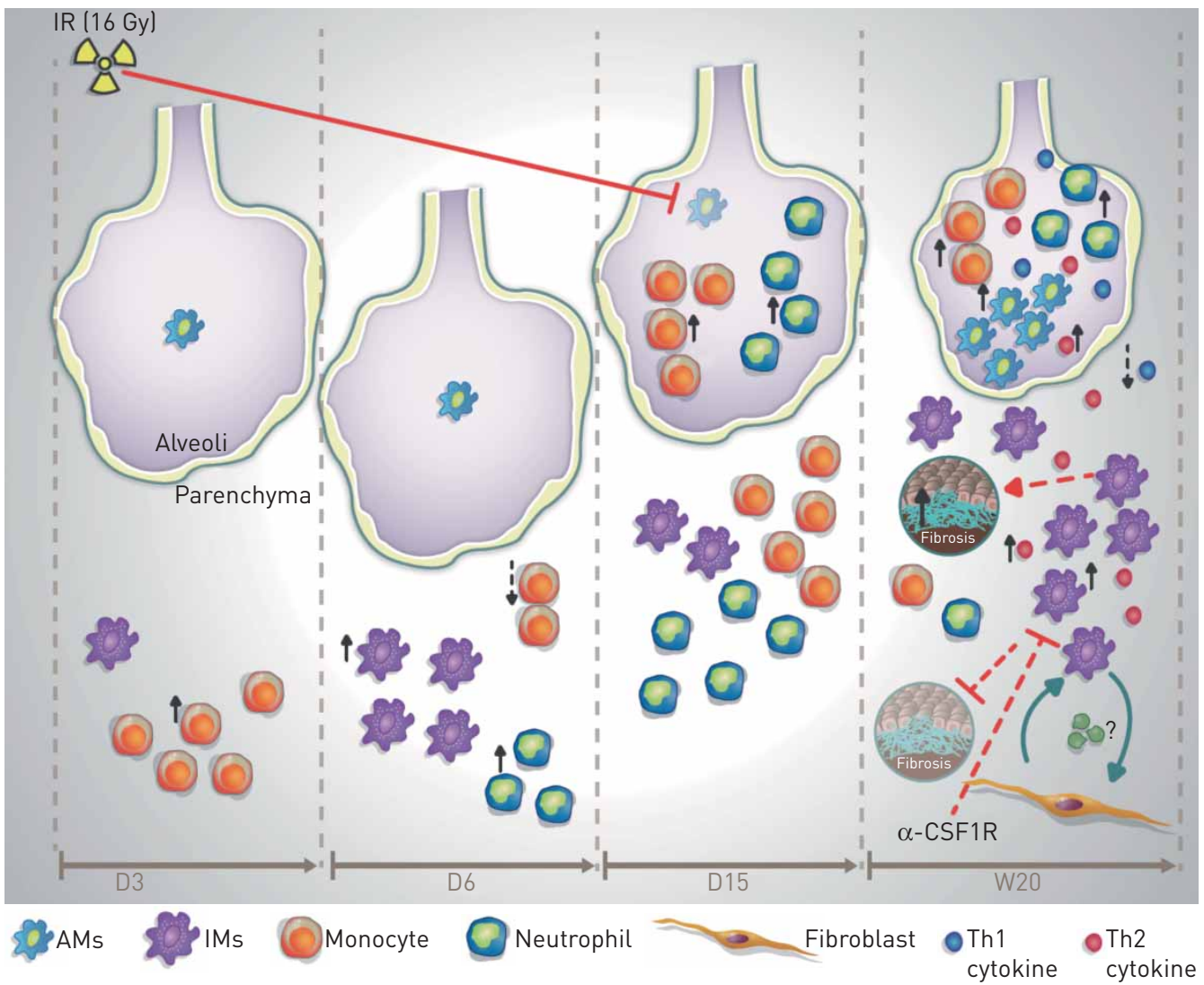

FIGURE 8 Proposed model of pulmonary macrophage contribution to radiation-induced lung fibrosis (RIF) During the acute phase response to a high dose of radiation (IR; $16 \mathrm{~Gy}$ ), an inflammatory response within parenchyma represented by an increase of monocyte number is observed at day (D) 3 after irradiation, followed by an increased number of neutrophils and pro-inflammatory tissue-infiltrating macrophages (IMs) at D6 after irradiation. At D15 the number of monocytes, neutrophils and IMs returns to the basal rate. At this time point monocyte and neutrophil number increases within the alveolar space accompanied by an important depletion of alveolar macrophages (AMs). During the late phase lup to week (W)20), there is a decrease in alveolar area and an increase of interstitial RIF score. In the alveolar space, increased AM number is associated with an increased secretion of T-helper cell type 1 (Th1) and Th2 cytokines and an increased number of neutrophils and monocytes. In the interstitial space, secretion of Th2 cytokines such as TIMP-1 is associated with increased IM number, which are able to induce direct activation of fibroblasts and vice versa. This interplay between IMs and fibroblasts contributes to pathogenesis of RIF, and points out the profibrotic role of activated IMs, while activated AMs seem to be non-profibrotic. Specific depletion of IMs using anti-CSF1R induces significant depletion of activated IMs, but not AMs, and reduces the fibrosis score.

prostate and breast cancer [30]. Thus, our results open interesting therapeutic perspectives, which can soon be evaluated in early-phase clinical trials.

In summary, despite recent advances in radiation oncology with treatment planning and delivery of image-guided radiation therapy, normal tissue toxicity is still a dose-limiting factor for optimal local tumour control. Additionally, as the number of long-term cancer survivors increases, severe complications emerge and dramatically impair patient quality of life. Here we propose a novel approach to improve the clinical management of radiation-induced complications via CSF1R inhibition and the depletion of activated interstitial macrophages.

\section{Acknowledgements}

The authors would like to thank Yann Lécluse, Philippe Rameau, Sophie Salome-Desnoulez (Imaging and Cytometry Platform (PFIC)) and Olivia Bawa (Preclinical Evaluation Platform (PFEP)) at Gustave Roussy (Villejuif, France) for their technical assistance; David Schaer (Eli Lilly, New York, NY, USA) for the helpful discussions. The authors also wish to thank Y. Julé and J.C. Gilhodes (Biocellvia, Marseille, France), for performing the automated histological image analyses of lung fibrosis and for the helpful discussions.

Author contributions: L. Meziani designed and performed experiments, conducted the data/statistical analysis and wrote the manuscript; M. Mondini designed anti-CSF1R experiments, supervised the experiments and wrote the manuscript; B. Petit performed experiments; A. Boissonnas analysed and interpreted the data; O. Mercier and 
V. Thomas de Montpreville collected human samples and reviewed the work; M-C. Vozenin and E. Deutsch designed and supervised experiments, provided funding and wrote the manuscript.

\section{References}

$1 \quad$ Yarnold J, Brotons MC. Pathogenetic mechanisms in radiation fibrosis. Radiother Oncol 2010; 97: 149-161.

2 Friedman SL. Preface. Hepatic fibrosis: pathogenesis, diagnosis, and emerging therapies. Clin Liver Dis 2008; 12: xiii-xxiv.

3 Ikushima H, Miyazono K. TGF $\beta$ signalling: a complex web in cancer progression. Nat Rev Cancer 2010; 10 415-424.

4 Wynn TA, Barron L. Macrophages: master regulators of inflammation and fibrosis. Semin Liver Dis 2010; 30: 245-257.

5 Belliere J, Casemayou A, Ducasse L, et al. Specific macrophage subtypes influence the progression of rhabdomyolysis-induced kidney injury. J Am Soc Nephrol 2015; 26: 1363-1377.

$6 \mathrm{Fu} \mathrm{CL}$, Odegaard JI, Hsieh MH. Macrophages are required for host survival in experimental urogenital schistosomiasis. FASEB J 2015; 29: 193-207.

7 Pyonteck SM, Akkari L, Schuhmacher AJ, et al. CSF-1R inhibition alters macrophage polarization and blocks glioma progression. Nat Med 2013; 19: 1264-1272.

8 Mok S, Tsoi J, Koya RC, et al. Inhibition of colony stimulating factor-1 receptor improves antitumor efficacy of BRAF inhibition. BMC Cancer 2015; 15: 356

9 De Palma M, Lewis CE. Macrophage regulation of tumor responses to anticancer therapies. Cancer Cell 2013; 23: 277-286.

10 Meng Y, Beckett MA, Liang $\mathrm{H}$, et al. Blockade of tumor necrosis factor $\alpha$ signaling in tumor-associated macrophages as a radiosensitizing strategy. Cancer Res 2010; 70: 1534-1543.

11 Klug F, Prakash H, Huber PE, et al. Low-dose irradiation programs macrophage differentiation to an iNOS $/ \mathrm{M}^{+}$ phenotype that orchestrates effective T cell immunotherapy. Cancer Cell 2013; 24: 589-602.

12 Travis EL. The sequence of histological changes in mouse lungs after single doses of X-rays. Int J Radiat Oncol Biol Phys 1980; 6: 345-347.

13 Wynn TA. Fibrotic disease and the $\mathrm{T}_{\mathrm{H}} 1 / \mathrm{T}_{\mathrm{H}} 2$ paradigm. Nat Rev Immunol 2004; 4: 583-594.

14 Chamoto K, Gibney BC, Ackermann M, et al. Alveolar macrophage dynamics in murine lung regeneration. J Cell Physiol 2012; 227: 3208-3215.

15 Zaynagetdinov R, Sherrill TP, Kendall PL, et al. Identification of myeloid cell subsets in murine lungs using flow cytometry. Am J Respir Cell Mol Biol 2013; 49: 180-189.

16 Thepen T, Van Rooijen N, Kraal G. Alveolar macrophage elimination in vivo is associated with an increase in pulmonary immune response in mice. J Exp Med 1989; 170: 499-509.

17 Tiemessen MM, Jagger AL, Evans HG, et al. $\mathrm{CD} 4^{+} \mathrm{CD} 25^{+} \mathrm{Foxp} 3^{+}$regulatory $\mathrm{T}$ cells induce alternative activation of human monocytes/macrophages. Proc Natl Acad Sci USA 2007; 104: 19446-19451.

18 Gilhodes JC, Julé Y, Kreuz S, et al. Quantification of pulmonary fibrosis in a bleomycin mouse model using automated histological image analysis. PLoS One 2017; 12: e0170561.

19 Morrison C. Immuno-oncologists eye up macrophage targets. Nat Rev Drug Discov 2016; 15: 373-374.

20 Rubin P, Casarett GW. Clinical radiation pathology as applied to curative radiotherapy. Cancer 1968; 22: 767-778.

21 Fajardo LF. Pathology of Radiation Injury. New York, Masson Pub., 1982.

22 Chandler DB, Bayles G, Fuller WC. Prostaglandin synthesis and release by subpopulations of rat interstitial macrophages. Am Rev Respir Dis 1988; 138: 901-907.

23 Gibbons MA, MacKinnon AC, Ramachandran P, et al. Ly6Chi monocytes direct alternatively activated profibrotic macrophage regulation of lung fibrosis. Am J Respir Crit Care Med 2011; 184: 569-581.

24 Beider K, Bitner H, Leiba M, et al. Multiple myeloma cells recruit tumor-supportive macrophages through the CXCR4/CXCL12 axis and promote their polarization toward the M2 phenotype. Oncotarget 2014; 5: 11283-11296.

25 Tripathi C, Tewari BN, Kanchan RK, et al. Macrophages are recruited to hypoxic tumor areas and acquire a pro-angiogenic M2-polarized phenotype via hypoxic cancer cell derived cytokines Oncostatin M and Eotaxin. Oncotarget 2014; 5: 5350-5368.

26 Comito G, Giannoni E, Segura CP, et al. Cancer-associated fibroblasts and M2-polarized macrophages synergize during prostate carcinoma progression. Oncogene 2014; 33: 2423-2431.

27 Wynn TA. Integrating mechanisms of pulmonary fibrosis. J Exp Med 2011; 208: 1339-1350.

$28 \mathrm{Xu}$ J, Escamilla J, Mok S, et al. CSF1R signaling blockade stanches tumor-infiltrating myeloid cells and improves the efficacy of radiotherapy in prostate cancer. Cancer Res 2013; 73: 2782-2794.

29 Jinushi M, Komohara Y. Tumor-associated macrophages as an emerging target against tumors: creating a new path from bench to bedside. Biochim Biophys Acta 2015; 1855: 123-130.

30 Smyth MJ, Ngiow SF, Ribas A, et al. Combination cancer immunotherapies tailored to the tumour microenvironment. Nat Rev Clin Oncol 2016; 13: 143-158. 